\title{
Éditorial: Énoncé de politique de l'Association canadienne de gérontologie sur les soins à domicile au Canada
}

Énoncé du problème: Le Canada n'a pas de politique ou de programme universel et global sur les soins à domicile.

Portée: Les Canadiens ne jouissent pas en ce moment de soins à domicile universellement accessibles; ces soins ne font pas partie du régime d'assurance-maladie. Les services offerts, les critères d'admissibilité et la tarification - importance et existence - varient d'une province à l'autre. Et pourtant beaucoup de personnes estiment que les soins à domicile constituent une composante nécessaire du système de soins de santé intégré. Le Forum national sur la santé (1997), le Conseil consultatif national sur le troisième âge (1995), l'Association canadienne des soins à domicile (1996) et les Infirmières de l'Ordre de Victoria du Canada (1997) ont tous recommandé que les services de santé et de soutien communautaires complets soient reconnus comme des services essentiels dans le système de santé canadien.

En effet, la réforme des soins de santé des années 1990 dans presque toutes les provinces (Mhatre \& Deber, 1992) ne fait-elle pas des soins à domicile la pierre angulaire du système complet de soins qu'il convient d'assurer aux personnes âgées et aux personnes de tous les âges.

Analyse des faits: Les partisans de l'élargissement des services de soins à domicile soutiennent que ces services sont opportuns et de bon rapport coût-efficacité. Les études sur l'optimisation des coûts ne sont pas concluantes. Nombre des premières études sur le sujet faites aux Etats-Unis (voir Chappel, 1994 pour une revue de ces études) constatent que les services de soins à domicile ne sont pas nécessairement plus efficaces par rapport au coût, bien que les personnes en bénéficiant disent quils améliorent leur qualité de vie. Une des difficultés que posent ces études tient au fait qu'elles ne distinguent pas les différentes fonctions des soins à domicile: substitution aux soins de courte durée en milieu hospitalier; substitution aux soins de longue durée en établissement; et fonction de maintien et de prévention. Certaines études concluent que les soins à domicile sont rentables à condition qu'ils ne ciblent que les personnes qui auraient été mises dans un établissement. Weissert (1985), défenseur bien 
connu de la position que les soins à domicile ne sont pas moins coûteux que les soins de longue durée dans un établissement, a récemment soutenu qu'ils peuvent l'être (Weissert et al., 1997). Les chercheurs commencent à peine à examiner cette question de manière plus sophistiquée, plus particulièrement depuis que Santé Canada a créé le Fonds pour l'adaptation des services de santé (FASS). Un récent rapport de la Saskatchewan (HSURC, 1998) conclut que les soins à domicile peuvent se substituer aux soins de courte durée en milieu hospitalier; que les patients subissant un traitement médical ou chirurgical peuvent recevoir des soins alternatifs, surtout des soins à domicile, qui donneront les mêmes résultats à coût moindre. Pour que les soins à domicile servent à une telle fin, il faut que les malades sortent de l'hôpital au moment indiqué. Il fait peu de doute, semble-t-il, que les services de soins à domicile peuvent être rentables, la question est de savoir dans quelles circonstances et pour quel niveau de soins requis. Les aspects qualité de vie et opportunité des soins ne sont pour ainsi dire pas mis en cause - les gens préfèrent être dans un milieu familier, donc chez eux, lorsqu'ils sont malades.

Les faits suggèrent aussi que les soins à domicile sont sous-financés. En 1997-1998, à peine plus de 2 millions de dollars provenant des fonds publics ont été consacrés aux soins à domicile au Canada (Santé Canada, 1998); cela ne représentait que 4 pour cent de la totalité des dépenses publiques pour les soins de santé. On estime en outre qu'environ 90 pour cent des services de soins à domicile sont financés par des fonds publics (Sorochan, 1995). Si les budgets des soins à domicile augmentent d'un bout à l'autre du pays, les fonds additionnels qui y sont affectés n'égalent pas l'augmentation de la demande de soins postérieurs aux soins de courte durée résultant de la restructuration des soins de santé et la sortie plus hâtive des hôpitaux de soins de courte durée. Les soins de longue durée aux malades chroniques assurés par des services communautaires ne sont pas que menacés de diminuer, ils diminuent réellement. (Home Support Administrators of Greater Victoria and the Islands, 1998). En d'autres termes, il existe des motifs réels d'inquiétude concernant le sous-financement des soins à domicile au Canada.

Enfin, nous savons qu'entre 70 et 80 pour cent des soins personnels offerts aux personnes âgées proviennent de réseaux informels constitués de la famille et des amis (Kane \& Kane, 1985). La restructuration des soins de santé, qui s'est accompagnée de fermetures de lits d'hôpitaux et, à certains endroits, de fermetures d'hôpitaux, de sorties plus rapides des hôpitaux, d'une augmentation des chirurgies ambulatoires et d'une restriction sur la construction d'établissements de soins de longue durée, mais pas d'une augmentation comparable du financement des soins à domicile, a accru le fardeau que porte les soignants informels. On doute que les soignants puissent fournir plus de soins qu'ils ne font déjà, soins qu'ils procurent à un coût considérable pour eux (RIS MRC CFAS, 1998). Nous 
savons que l'absence de soignants informels est le plus grand prédicteur de soins de longue durée en établissement pour les personnes âgées (Shapiro \& Tate, 1988) et que les soins à domicile peuvent prolonger les soins informels et prévenir l'épuisement des personnes assurant ces soins (Horowitz \& Dobrof, 1982; Chappell, 1992).

Recommandation: L'Association canadienne de gérontologie recommandent que les gouvernements fédéral, provinciaux et territoriaux s'appliquent sans délai à mettre sur pied un programme de soins à domicile complets et accessibles à tous les Canadiens.

Ce programme doit:

a) Comporter un financement adéquat de la formation, de la prestation des services et de l'évaluation;

b) Comporter des normes nationales de formation, de prestation de services et d'évaluation;

c) Offrir les services indiqués aux soignants informels.

\section{Références}

Chappell, N.L. (1992). Social support and aging. Butterworths Canada Ltd.

Chappell, N.L. (1994). Home care research: What does it tell us? The Gerontologist, 34(1), 116-120.

Health Services Utilization and Research Commission (HSURC). (Mars 1998). Hospital and home care study.

Home Support Administrators of Greater Victoria and the Islands. (Mai 1998). Home support services in capital health region: A utilization study, 1989-1998.

Horowitz, A., \& Dobrof, R. (1982). The role of families in providing long-term care to the frail and chronically ill elderly living in the community. Health Care Financing Administration, USDHHS Grant No. 18-P-97541/2-02.

Kane, R.A., \& Kane, R.L. (1985). Feasibility of universal long-term-care benefits: ideas from Canada. New England Journal of Medicine, 312(21), 1357-1364.

Mhatre, S.L., \& Deber, R.B. (1992). From equal access to health care to equitable access to health: A review of Canadian provincial health commissions and reports. International Journal of Health Services, 22(4), 645-668.

RIS MRC CFAS (Resource implications study of Medical Research Council Cognitive Function and Ageing Study) (1998). Mental and physical frailty in older people: the costs and benefits of informal care. Ageing and Society, 18, $317-354$.

Santé Canada. (1998). Fonds publics consacrés aux soins à domicile au Canada de 1975-1976 à 1997-1998. Feuillets d'information.

Shapiro, E., \& Tate, R. (1988). Who is really at risk of institutionalization? The Gerontologist, 29(2), 237-245.

Sorochan, M. (Janvier 1995). Home care in Canada. Caring Magazine.

Weissert, W.G. (1985). Seven reasons why it is so difficult to make communitybased long term care cost-effective. Health Services Research, 20(4), 423-433. 
Weissert, W.G., Lesnick T., Musliner, M., \& Foley, K.A. (1997). Cost savings from home and community-based services: Arizona's capitated Medicaid longterm care program. Journal of Health Politics, Policy \& Law, 22(6), 1329-1357.

Rédigé par Neena L. Chappell, Ph.D., Directrice du Centre on Aging, University of Victoria, à la demande du conseil d'administration de l'Association canadienne de gérontologie, l'exposé ci-dessus constitue la position officielle du conseil adoptée en avril 1999. 